\title{
Full Phononic Bandgap in 2D-Optomechanical Crystals
}

\author{
T. P. Mayer Alegre, A. H. Safavi-Naeini, M. Winger, and O. Painter \\ Thomas J. Watson, Sr., Laboratory of Applied Physics, California Institute of Technology, Pasadena, CA \\ 91125, USA. \\ e-mail: talegre@caltech.edu
}

\begin{abstract}
We demonstrate simultaneous strong confinement and interaction of photons and phonons in a quasi two-dimensional (2D) slab.

(c) 2010 Optical Society of America

OCIS codes: (220.4880) Optomechanics; (230.5298) Photonic crystals; (350.4238) Nanophotonics and photonic crystals
\end{abstract}

In direct analogy to periodic potentials for electrons in crystals and photons in photonic crystals, periodicity in elastic structures gives rise to interesting and useful phenomena for phonons. Due to their ability to control acoustic wave propagation with phononic bandgaps, these structures, known as phononic crystals, have received much attention in both science and technology. Although phononic bandgaps have been experimentally shown in a variety of systems and dimensionalities, few have considered them in geometries amenable to phononic and optomechanical circuitry. Perhaps the most promising approach is that of optomechanical crystals (OMCs) [1], which place phonons and photons on equivalent footing and allow for the unification of photonic and phononic crystal circuitry. Consequently, the extension of OMCs to 2D geometries (2DOMC) has become the back-bone of proposals utilizing optomechanical effects to delay, store, process, and convert light and sound [2,3]. Here, we experimentally demonstrate a 2D platform for optomechanics by achieving strong phonon-photon confinement and interaction in a cavity based on a quasi-2D phononic crystal defect. In addition to making steps towards realizing more complex 2DOMC systems, we illustrate a sideband resolved optomechanical system with promising applications in classical and quantum information processing, measurement, and the study of fundamental dissipative processes in nanomechanics. As our phononic crystal
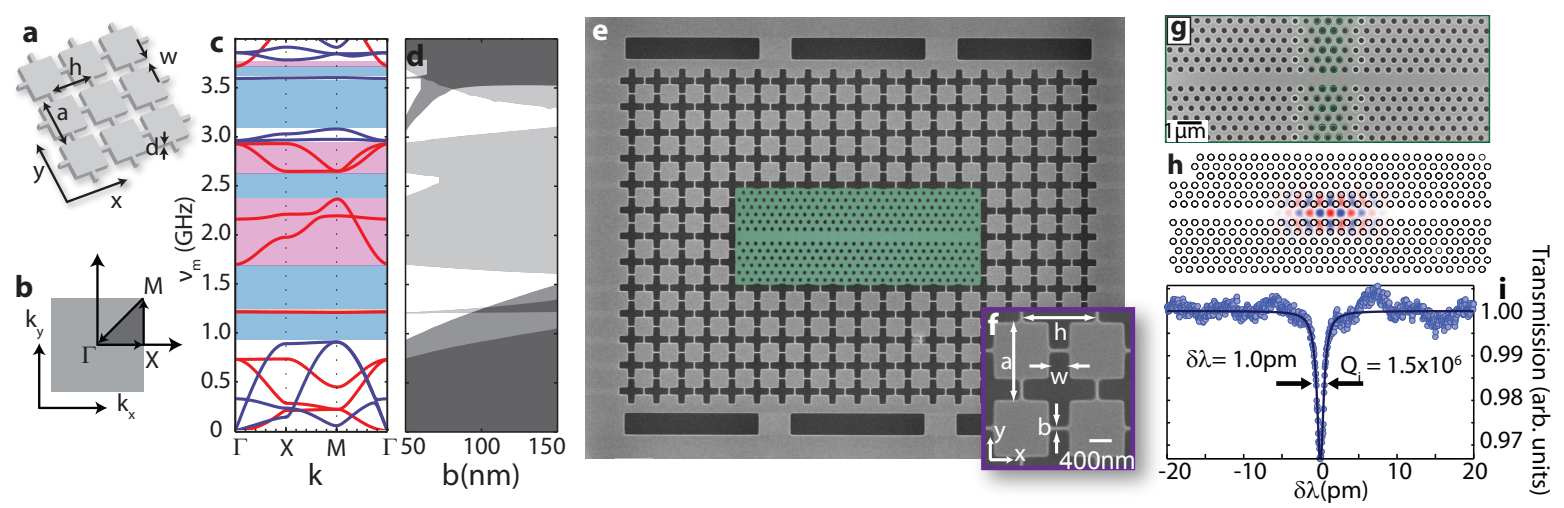

Fig. 1. a, representation of the real space crystal lattice with lattice constant $a$, cross length $h$, cross width $w$, and membrane thickness $d$. The bridge width is defined as $b=a-h$. $\mathbf{b}$, reciprocal lattice of the first Brillouin zone for the phononic crystal. $\mathbf{c}$, phononic band diagram for the nominal structure with $a=1.265 \mu \mathrm{m}, h=1.220 \mu \mathrm{m}, w=340 \mathrm{~nm}$. Dark blue (red) lines represent the bands with even (odd) vector symmetry for reflections about the $x-y$ plane. d, tuning of the band gap with the bridge width $b$. White areas correspond to regions of full band gaps. e, scanning electron micrograph (SEM) of one of the fabricated structures false colored (green) on the photonic regions. f, dimensions of the fabricated phononic crystal. g, SEM of the optical heterostructure within the phononic crystal. $\mathbf{h}$, finite element (FEM) simulation of $E_{\mathrm{y}}$ (y-component electrical field) for the optical cavity. $\mathbf{i}$, measured transmission spectrum of the optical cavity.

we use the "cross" structure proposed theoretically in [4] and schematically shown in Fig. 1(a). Geometrically, the structure consists of an array of squares connected to each other by thin bridges. A typical band diagram for a nominal structure is shown in Fig. 1(c). The symmetry-dependent bandgap (shaded) for the even modes of the simulated cross structure extends from $0.91 \mathrm{GHz}$ to $3.6 \mathrm{GHz}$. To achieve strong co-localization of phonons and photons, we embed a photonic crystal heterostructure cavity [5] (green area) within a phononic crystal, as shown in Fig. 1(e). In effect, the embedded optical cavity plays the role of a defect in a phononic crystal, in which the mechanical excitations of the structure may be localized. Such a phonon-photon heterostructure design allows for nearly independent tuning of the mechanical and optical properties of our system, despite the strong co-localization. The fabricated device, defined in a $220 \mathrm{~nm}$ thick silicon membrane, is shown in Fig. 1(e). By varying the bridge width $b$ of the phononic crystal, we cause the lower frequency bandgap boundary to tune to higher frequencies, while the mechanical modes are affected only marginally (see Fig. 1(d) and Fig. 2(a)). This allows us to deterministically shift the bandgap edge across our 

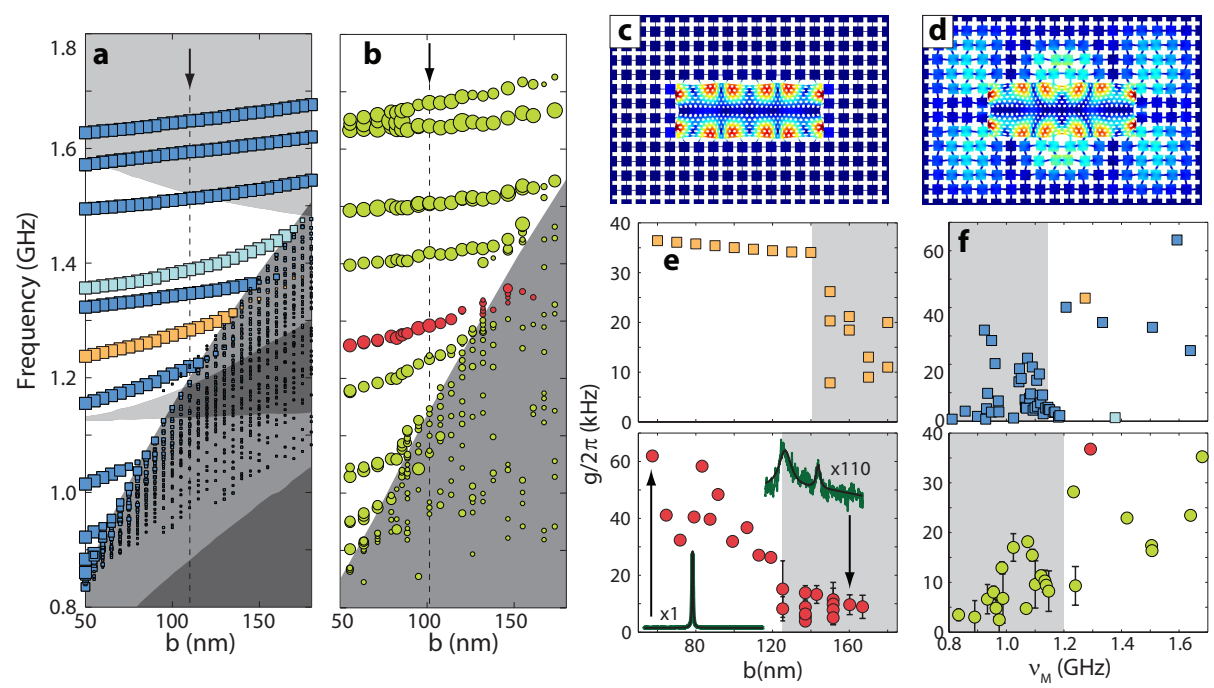

Fig. 2. a, results of full 3D-FEM simulations of phononic localized modes using absorbing perfect-matched-layers (PML) to account for $Q_{\mathrm{M}}^{(\mathrm{rad})}$. Each square corresponds to a single mode, where its size is proportional to the logarithm of $Q_{\mathrm{M}}^{(\mathrm{rad})}$. The gray shaded squares represent modes which are optically dark. b, measurement (circles) results of the localized mechanical modes. The marker sizes are proportional to measured $Q_{\mathrm{M}}$. c (d), FEM simulations of the displacement field amplitude $|\mathbf{Q}(\mathrm{r})|$ for the mechanical mode within the bandgap (on edge of the bandgap) at $1.35 \mathrm{GHz}$. Coupling of these localized photon and phonon modes shown here are computed to yield an optomechanical coupling length of $L_{\mathrm{OM}}=6.1 \mu \mathrm{m}$ with a motional mass of $m_{\mathrm{eff}}=3.3 \mathrm{pg}$, corresponding to a single-phonon coupling rate of $g=2 \pi \times 67 \mathrm{kHz}$. e simulated (squares) and measured (circles) optomechanical coupling rate $g$ extracted from the series of spectra for the highlighted mechanical mode shown on figures $\mathbf{a}$ and $\mathbf{b}$ respectively. $\mathbf{f}$ same as $\mathbf{e}$ for the device highlighted with the dashed line on $\mathbf{a}$ and $\mathbf{b}$.

mechanical modes, and observe, through simulation and experiment, the changes in their lifetime, density of states, and localization, as signatures of a phononic bandgap. To probe the mechanical modes we take advantage of the large optomechanical coupling in these structures. The optical modes of the devices are probed with a tapered optical fiber in the near-field of the defect cavity, simultaneously sourcing the cavity field and collecting the transmitted light in a single channel. The transmitted light through the cavity is modulated by the thermally excited mechanical motion of the modes localized in the vicinity of the optical cavity, generating a series of optical sidebands. The optical signal is sent into a high speed photodetector, and the microwave spectrum measured by a high frequency oscilloscope. Fig. 2(a) and (b) show the simulated and measured mechanical modes as a function of the bridge size $b$, with the marker size proportional to the mechanical quality factor. We can easily identify the lower boundary of the full band gap region (gray shaded area) by the abrupt change in the lifetime (or the mechanical quality factor), and the available mechanical density of states. The mechanical mode localization is also affected by the presence of the band gap as seen on Fig. 2(c) and Fig. 2(d). The change in the confinement is further tested by simulating and measuring the change of the optomechanical coupling when a given mode crosses the band edge (Fig. 2(e)). At room temperature, most of the localized phonon resonances inside the phononic bandgap were found to have mechanical $Q_{\mathrm{M}} \mathrm{s}$ of approximately 1000-3000. In the absence of clamping losses, other forms of damping, such as temperature-dependent thermoelastic damping (TED) [6], begin to dominate. By measuring the transduced thermal motion for a bandgap-localized phonon mode, we find that the resonances do substantially narrow at low temperatures (not shown), achieving a maximum $Q_{\mathrm{M}}=1.3 \times 10^{4}$ at $T=10 \mathrm{~K}$, indicating that the mechanical damping rate is not limited by clamping but by temperature-dependent loss channels such as TED.

\section{References}

1. M. Eichenfield, J. Chan, R. M. Camacho, K. J. Vahala, and O. Painter, "Optomechanical crystals," Nature 462, 78-82 (2009).

2. D. Chang, A. H. Safavi-Naeini, M. Hafezi, and O. Painter, "Slowing and stopping light using an optomechanical crystal array," 1006.3829 (2010).

3. A. H. Safavi-Naeini and O. Painter, "Proposal for an Optomechanical Traveling Wave Phonon-Photon Translator," 1009.3529 (2010), to appear in New J. Phys.

4. A. H. Safavi-Naeini and O. Painter, "Design of optomechanical cavities and waveguides on a simultaneous bandgap phononic-photonic crystal slab," Optics Express 18, 14 926-14943 (2010).

5. B.-S. Song, S. Noda, T. Asano, and Y. Akahane, "Ultra-high-Q photonic double-heterostructure nanocavity,” Nat Mater 4, 207-210 (2005).

6. R. Lifshitz and M. L. Roukes, "Thermoelastic damping in micro- and nanomechanical systems," Phys. Rev. B 61, 5600-5609 (2000). 